\section{DGAT out of the bag}

\section{By Tracey Baas, Senior Editor}

U.S. and French researchers have shown that DGAT1, an enzyme involved in lipid droplet biogenesis, is a host factor for HCV infection. ${ }^{1}$ The results have prompted Via Pharmaceuticals Inc. to consider repurposing its DGAT1 inhibitors, which are in preclinical development for metabolic indications.

The findings have their roots in office location and serendipity. Lead principal investigators Melanie Ott, associate investigator at the University of California, San Francisco's Gladstone Institute of Virology and Immunology, and Robert Farese Jr., senior investigator at the Gladstone Institute of Cardiovascular Disease, had nearby offices and saw a chance to work together on something that required their respective areas of expertise: virology and cellular lipid metabolism.

Building on 2007 findings $^{2}$ that showed lipid droplets are involved in $\mathrm{HCV}$ production, the Gladstone researchers hypothesized that enzymes responsible for lipid droplet biogenesis-diacylglycerol O-acyltransferases (DGATs) - also could play a role in the virus' life cycle. Serendipitously, the Farese lab had a small molecule DGAT1 (diacylglycerol O-acyltransferase-1) inhibitor on hand that had been developed for clinical trials for metabolic disease.

Ott and Farese teamed up with researchers at University Paris Descartes to test their hypothesis.

In cultured human hepatoma cells and primary human hepatocytes, the inhibitor decreased the production of infectious viral particles compared with vehicle.

Surprisingly, DGAT1 inhibition did not lead to an apparent decrease in lipid droplet biogenesis, suggesting that reductions in lipid droplets were not responsible for the inhibitory effect on $\mathrm{HCV}$. Indeed, neither the DGAT1 inhibitor nor anti-DGAT1 small hairpin RNA changed the overall lipid content or lipid droplet numbers in hepatoma cells.

Rather, in vitro studies showed that inhibiting DGAT1 caused the HCV core protein to remain in the endoplasmic reticulum of hepatoma cells instead of associating with lipid droplets, thus halting the life cycle of the virus.

Together, the data demonstrate that DGAT1 directly interacts with the HCV core protein and transports it to DGAT1-generated lipid droplets, thereby facilitating virus assembly and maturation (see Figure 1, "Model of HCV core recruitment to DGAT1-generated lipid droplets").

Data were published in Nature Medicine.

\section{Playing host}

John McLauchlan, associate director of the Medical Research Council-University of Glasgow Centre for Virus Research, said the findings "establish proof of principle that DGAT1 inhibitors could be

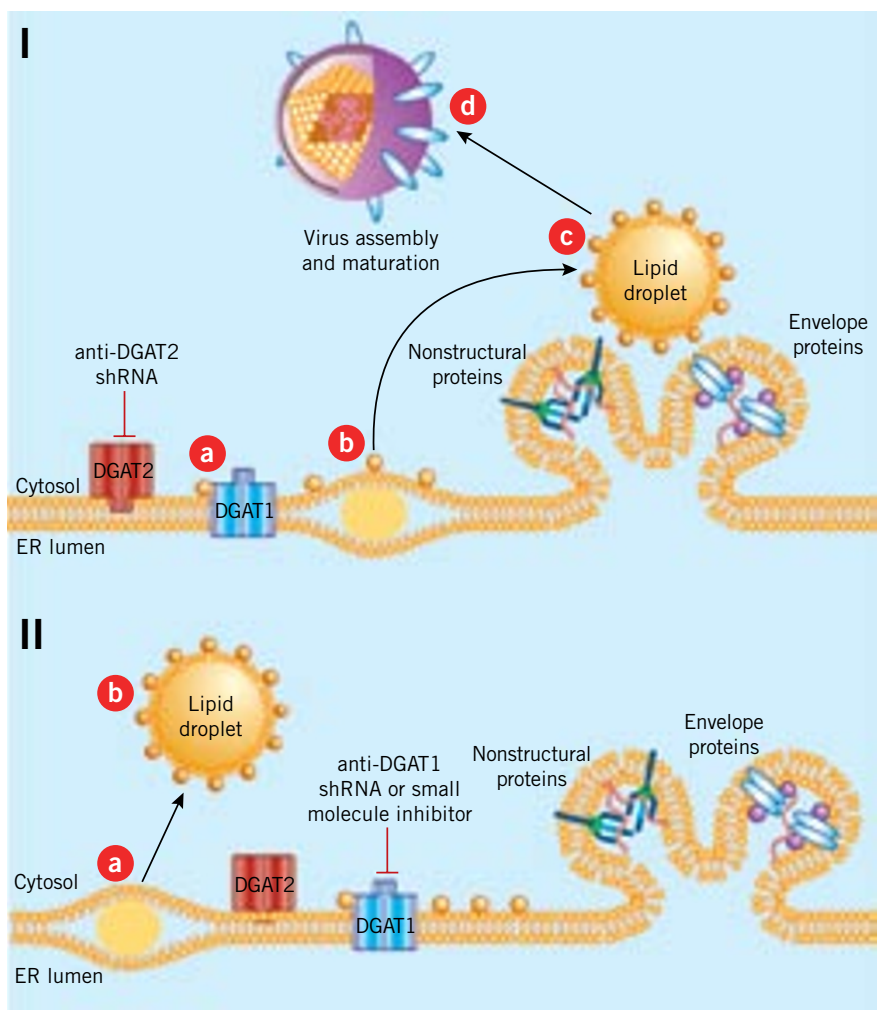

Figure 1. Model of HCV core recruitment to DGAT1-generated lipid droplets. Diacylglycerol O-acyltransferase-1 (DGAT1) and DGAT2 enzymes help to catalyze triglyceride biosynthesis and are essential in lipid droplet production.

(I) The HCV core protein interacts with DGAT1 [a] at the endoplasmic reticulum (ER) and thereby gains access to developing DGAT1-generated lipid droplets [b]. This step is crucial to recruit HCV RNA replication complexes to ER membranes in the vicinity of lipid droplets [c] and to initiate assembly and maturation of progeny virions [d]. Small hairpin RNA that targets DGAT2 does not disrupt HCV production.

(II) siRNA or small molecule inhibitors that target DGAT1 prevent the formation of DGAT1-generated lipid droplets and disrupt the translocation of the HCV core protein to lipid droplets [a]. Infectious HCV particles cannot form despite the presence of DGAT2-generated droplets [b].

a therapeutic route for treating chronic infection. Clearly, there are mechanisms in virus assembly that as yet have not been discovered, and identifying a role for DGAT1 represents an additional part of the jigsaw."

HCV-host interactions represent attractive targets for antiviral development because it is more difficult for the virus to develop escape mutations against therapeutics that target host cell factors.

"I would be fascinated to see DGAT1 inhibitors used for an antiHCV indication," said Paul Targett-Adams, principal scientist of the infectious diseases group at Pfizer Inc. "Targeting a host cell-encoded, 
rather than a virus-encoded, function has an advantage: the genetic barrier to resistance is generally higher and it is harder for the virus to mutate and escape the inhibitory conditions."

According to McLauchlan, "advancing the study towards use of this approach in the clinic would require analysis in other systems that may support the in vitro findings, such as relevant animal models."

Ott said her group plans to do just that. "In collaboration with colleagues at UCSF and the Salk Institute for Biological Studies, we are planning to continue our work in humanized mice, produced using a hepatic xenorepopulation system" developed by Markus Grompe, a professor of molecular and medical genetics at the Oregon Health \& Science University. ${ }^{3}$

Because HCV doesn't infect mouse hepatocytes, the system uses chimeric mice that have livers repopulated by human hepatocytes.

Ott's main focus is on HCV but she thinks the strategy could perhaps be used to study other models of viral infection reliant on lipid droplets or membrane lipid rafts.

Farese said he's interested in seeing whether inhibiting acyl CoA:cholesterol acyltransferases (ACATs), another group of lipid dropletassociated enzymes, affects viruses that are dependent on lipid droplets to complete their life cycle. A handful of companies had developed ACAT inhibitors for atherosclerosis, but interest has waned due to disappointing results in clinical trials. ${ }^{4-6}$

Meanwhile, Via is thinking about the direction of its DGAT1 work based on the new findings.

Via's DGAT1-targeting molecules are being tested in animal models of obesity, diabetes and lipid triglyceride modulation. Based animal model work, CEO Lawrence Cohen and SVP of R\&D Rebecca Taub believe that DGAT1-targeting small molecules will show good drug levels in the liver. Via in-licensed the DGAT1 program from Roche at the end of 2008.
Targett-Adams did say that "modulation of DGAT1 activity may be sufficient for efficacious treatment of lipid-related disorders of humans, but total inhibition of DGAT1 activity may be required to eliminate $\mathrm{HCV}$ infection. There may be pharmacological hurdles to overcome to abrogate DGAT1 activity in humans for a period of time necessary to promote elimination of the virus."

UCSF has filed for a patent that covers targeting DGAT1 to treat HCV infection. The IP is available for licensing, and Ott said that discussions with undisclosed companies are ongoing.

Baas, T. SciBX 3(42); doi:10.1038/scibx.2010.1253

Published online Oct. 28, 2010

\section{REFERENCES}

1. Herker, E. et al. Nat. Med.; published online Oct. 10, 2010; doi: $10.1038 / \mathrm{nm} .2238$

Contact: Melanie Ott, The J. David Gladstone Institutes, San Francisco, Calif. e-mail: mott@gladstone.ucsf.edu

2. Miyanari, Y. et al. Nat. Cell Biol. 9, 1089-1097 (2007)

3. Azuma, H. et al. Nat. Biotechnol. 25, 903-910 (2007)

4. Tardif, J.-C. et al. Circulation 110, 3372-3377 (2004)

5. Nissen, S.E. et al. N. Engl. J. Med. 354, 1253-1263 (2006)

6. Farese, R.V. Jr. Arterioscler. Thromb. Vasc. Biol. 26, 1684-1686 (2006)

\section{COMPANIES AND INSTITUTIONS MENTIONED}

Gladstone Institute of Cardiovascular Disease, San Francisco, Calif. Gladstone Institute of Virology and Immunology, San Francisco, Calif. Medical Research Council-University of Glasgow Centre for Virus Research, Glasgow, U.K.

Oregon Health \& Science University, Portland, Ore.

Pfizer Inc. (NYSE:PFE), New York, N.Y.

Roche (SIX:ROG; OTCQX:RHHBY), Basel, Switzerland

Salk Institute for Biological Studies, La Jolla, Calif.

University of California, San Francisco, Calif.

University Paris Descartes, Paris, France

Via Pharmaceuticals Inc. (Pink:VIAP), San Francisco, Calif. 